\title{
Perfil de metadados OBAA utilizado no ensino de profissionais de saúde
}

\author{
Gustavo Schwarz
}

Universidade Federal do Rio Grande do Sul-gustavo.sne@gmail.com

Otávio Costa Acosta

Universidade Federal do Rio Grande do Sul - ocacosta@inf.ufrgs.br

\author{
Alexandre Moretto Ribeiro \\ Universidade Federal do Rio Grande do Sul - alexandremorettoribeiro@gmail.com
}

\section{Cecília Dias Flores}

Universidade Federal de Ciências da Saúde de Porto Alegre dfloresorama@gmail.com

\begin{abstract}
Resumo: $O$ presente trabalho apresenta a construção de um perfil de metadados para a utilização em um repositório de Objetos de Aprendizagem na área da saúde. Este perfil é criado juntamente com mecanismo de extensão que o OBAA herda do padrão IEEE-LOM. Para a criação deste perfil duas versões foram necessárias, sendo descrito o mapeamento de cada uma delas. Perfis de metadados do OBAA têm como finalidade principal reduzir a complexidade de gerencia de objetos de aprendizagem em determinado contexto, sendo a área da saúde o foco deste artigo.
\end{abstract}

Palavras-Chave: OBAA, UNASUS, perfil de metadados, objetos de aprendizagem

\begin{abstract}
This article presents the construction of a metadata profile for its usage in a Learning Objects repository in the subject of health. This profile is created together with an extension mechanism which the OBAA inherits from the IEEE-LOM standard. For the creation of this profile, two versions were necessary, and their mapping are described for each. OBAA metadata profiles have the main purpose of reducing the management complexity for learning objects in a specified context, with the health subject area being the focus of this article.
\end{abstract}

Keywords: OBAA, UNASUS, metadata profile, learning objects

\section{Introdução}

A transformação e evolução das tecnologias têm provocado grande impacto na sociedade, em especial a informática e a telecomunicação, também chamadas de tecnologias do conhecimento. (Ministério da Educação, 2007, p. 50-51). Neste novo cenário tecnológico os profissionais de educação devem procurar adquirir novas habilidades a fim de produzir e compartilhar novos conhecimentos. Além disso, a utilização de elementos multimídia, tais como simulações, imagens, jogos e textos desempenham um papel fundamental na aquisição do conhecimento se forem bem 
empregados. Estes recursos, também conhecidos com Objetos de Aprendizagem (OA), criam um novo modo de aprendizagem/ensino apoiada por computador. Desta forma $\mathrm{o}$ professor abandona o papel de transmissor de informação para desempenhar o papel de mediador do aprendizado (Behar; Gaspar, 2007, p. 3).

Os principais fatores que favorecem a utilização dos OAs são: flexibilidade, facilidade de atualização, customização e interoperabilidade. (Ministério da Educação, 2007, p. 20). Para atender estas características, é necessário que os OAs sejam descritos segundo um padrão de metadados (Gomes et al., 2005, p. 202). Sendo que alguns dos principais padrões utilizados ao redor do mundo são: LTSC, o ARIADNE, o IMS, o ADL, o LOM, o SCORM. No Brasil, está sendo proposto o padrão OBAA, partindo de uma demanda governamental, conforme edital MCT/FINEP/MC/FUNTTEL Plataformas para Conteúdos Digitais - 01/2007(Vicari et al., 2009).

Este trabalho apresenta alguns recursos referentes ao padrão OBAA, principalmente no que diz respeito à criação de perfis de metadados. Será descrito um perfil criado para a área da saúde. A seção 2 apresenta de forma resumida o padrão OBAA, a seção 3 apresenta a Universidade Aberta do SUS, a seção 4 mostra o mapeamento realizado entre os metadados do OBAA e do sistema UNASUS e o artigo é concluído com as considerações finais e os direcionamentos para trabalhos futuros.

\section{Projeto OBAA}

Em um mundo cada vez mais conectado, onde as pessoas têm acesso aos mais variados dispositivos e serviços digitais, como internet, dispositivos móveis e TV Digital é necessário haver uma padronização dos metadados (MDs) entre estas três plataformas, facilitando o gerenciamento da sobrecarga de informações. No presente artigo será abordado o padrão de MDs OBAA, que é um projeto aberto e que tem como principal objetivo possibilitar a utilização dos OAs em diferentes plataformas além de facilitar seu uso no contexto educacional brasileiro. (Vicari et al., 2009, p. 9).

OBAA, abreviação de Objetos de aprendizagem Baseados em Agentes Autônomos, é um projeto desenvolvido por pesquisadores da UFRGS em parceria com o FINEP, no qual foi criado um padrão de MDs que atenda os requisitos de portabilidade e que também facilitem o uso de recursos digitais no contexto educacional brasileiro. Ao elaborar tal padrão, tomou-se o cuidado de manter a compatibilidade com padrões internacionalmente já conhecidos, tais como o IEEE-LOM (IEEE Computer Society, 2005), IMS (IMS Global Learning Consortium, 2004), TV-Anytime (McParland, 2005) e o MPEG-7 (Værvågen; Dahl, 2007).

\subsection{Padrão de metadados OBAA}

Os MDs que compõem o padrão OBAA são uma extensão do IEEE-LOM, agregando informações de outros padrões internacionalmente conhecidos já citados anteriormente. Além disso, a criação de novos MDs foi necessária para permitir que os OAs possam ser facilmente utilizados no contexto educacional e tecnológico atual.

A versão 1 do OBAA possui um total de 164 MDs os quais estão divididos em 
11 grupos diferentes. Uma breve descrição de cada grupo pode ser vista a seguir (Vicari et al., 2009):

1. General: Corresponde à descrição geral do OA como um todo, possui informações como: nome, descrição, idioma do OA, entre outros. É baseado no padrão IEEE-LOM e possui 11 MDs.

2. LifeCycle: Contém informações sobre o histórico e o estado atual (versão) do OA. É baseado no padrão IEEE-LOM e possui 7 MDs.

3. MetaMetadata: Possui informações sobre os próprios MDs do OA, ou seja, informações adicionais sobre os próprios MDs. É baseado no padrão IEEELOM e possui 10 MDs.

4. Technical: Categoria que descreve os requisitos técnicos e características do objeto de aprendizagem. Neste grupo também são abordados MDs que definem serviços, ontologias, linguagens de conteúdo e protocolos de interação que estão associados ao objeto, visando tratar de questões de interoperabilidade com a futura web semântica. É baseado no padrão IEEELOM e possui 39 MDs, sendo 13 nativos do LOM e 26 do OBAA.

5. Educational: Categoria que descreve características educacionais ou pedagógicas do objeto de aprendizagem. É baseado no padrão IEEE-LOM e possui 19 MDs, sendo 12 nativos do LOM e 7 do OBAA.

6. Rights: Categoria com informações sobre propriedade intelectual e condições de uso do OA, tais como: se existe algum custo atribuído para que sua utilização seja possível; restrições de direitos autorais e; comentários e condições sobre o uso do OA. É baseado no padrão IEEE-LOM e possui 4 MDs.

7. Relation: Categoria que possibilita definir relacionamentos entre diferentes objetos de aprendizagem. É baseado no padrão IEEE-LOM e possui 7 MDs.

8. Annotation: Grupo com comentários adicionais sobre o uso educacional do OA. Contém observações sobre o uso pedagógico deste objeto e informações sobre quando e por quem as observações foram criadas. É baseado no padrão IEEE-LOM e possui 4 MDs.

9. Classification: Categoria que descreve onde o objeto de aprendizagem se encaixa em um sistema de classificação. É baseado no padrão IEEE-LOM e possui 9 MDs.

10. Accessibility: Categoria que reúne informações sobre os recursos de acessibilidade. É baseado no padrão IMS AcessForAll e possui 35 MDs.

11. SegmentInformationTable: Em muitos casos, é necessário fazer uma subdivisão lógica de um OA, permitindo a organização por assuntos ou módulos. Desta forma, o grupo SegmentInformationTable possui informações que possibilitam a segmentação de um determinado OA. É baseado nos padrões TV-Anytime e MPEG-7. Possui 19 MDs.

No padrão OBAA é possível criar diferentes tipos de perfis, onde um subconjunto de MDs é selecionado no próprio padrão, criando assim um conjunto mais 
reduzido de MDs, o que gera a redução na complexidade de gerenciamento dos recursos educacionais. "Os Perfis de Metadados (PM) são formados por subconjuntos dos MDs de OAs definidos na presente proposta de padrão." (Vicari et al., 2009, p. 45).

Alguns exemplos de perfis previamente definidos no padrão são o perfil Educacional que define um conjunto mínimo de metadados necessários para suportar aplicações educacionais básicas; e o perfil de Acessibilidade que define qual conjunto mínimo de metadados são necessários para aplicações com suporte a acessibilidades. (Vicari et al., 2009).

Cabe ressaltar que, além dos perfis supracitados, o OBAA é flexível ao ponto de permitir a criação de outros perfis, inclusive com metadados que não estejam presentes no padrão. Esta inclusão é realizada através do mecanismo de extensão que o OBAA herda do IEEE-LOM. Em Xavier (2009) é possível encontrar maiores detalhes de como realizar extensões de metadados utilizando o padrão LOM. A firgura a seguir busca representar estes dois tipos de perfis:

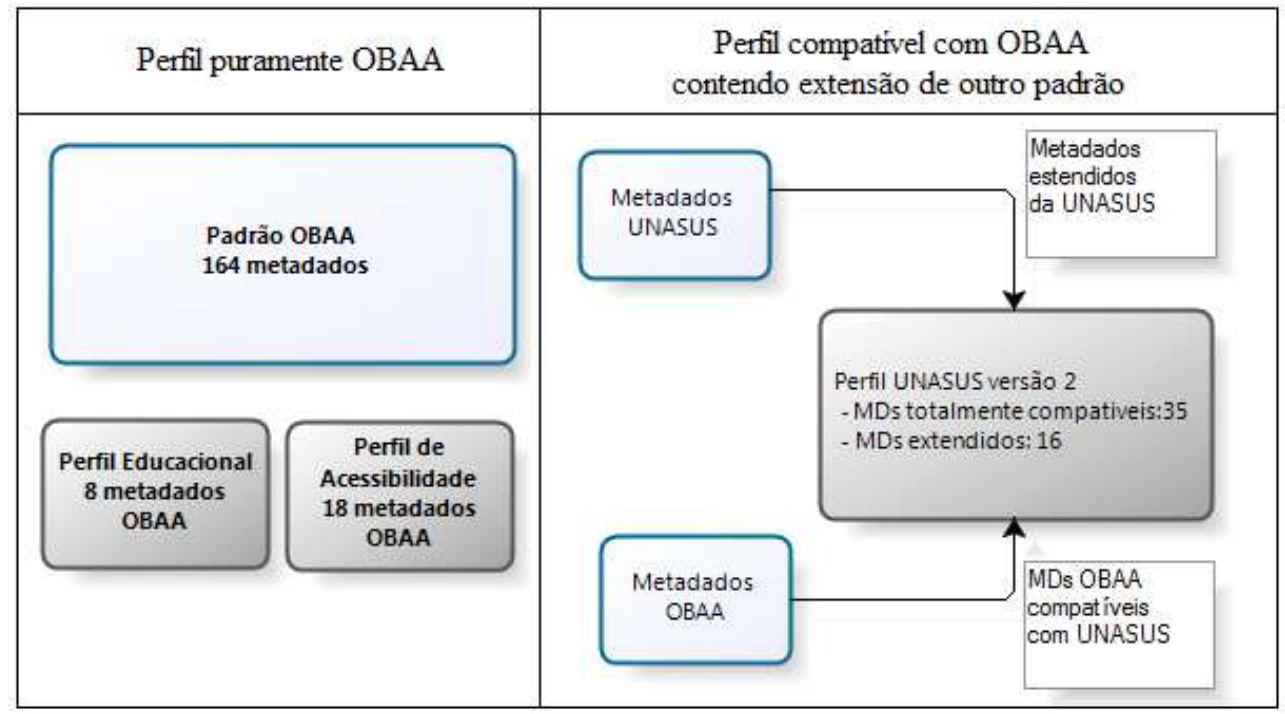

Figura 1: Perfis de metadados

\subsection{Repositório de Objetos de Aprendizagem OBAA}

No contexto do projeto OBAA MILOS/Comunidade encontra-se a criação de um repositório para facilitar a utilização dos OAs por professores e alunos. A ferramenta utilizada foi o DSpace (Duraspace, 2012). Não é o objetivo do presente artigo aprofundar-se neste tema, até mesmo porque este é um assunto para outro artigo. Atualmente o repositório possui 40 objetos submetidos com seus respectivos metadados, 5 comunidades e 5 coleções. Para acessá-lo deve-se utilizar o seguinte endereço: http://repositorio.portalobaa.org/.

\section{A utilização do padrão OBAA na UNASUS}

Uma das iniciativas para a utilização do projeto OBAA é a criação de um perfil de MDs junto à Universidade Aberta do Sistema Único de Saúde (UNASUS). Esta 
universidade foi criada com a finalidade de atender às necessidades de captação e educação permanente dos trabalhadores do SUS através de modalidades de educação a distância. Desta forma, médicos, odontólogos, enfermeiros, agentes comunitários de saúde, gestores, entre outros trabalhadores do SUS, poderão ter acesso de pós-graduação e extensão universitário. "A Universidade Aberta do SUS - UNASUS é uma iniciativa do Ministério da Saúde em parceria com estados, municípios, instituições públicas de ensino superior e organismos internacionais para oferta de cursos de pós-graduação e de extensão universitária." (UNASUS, 2011)

Visto o contexto da UNASUS, observa-se a necessidade de catalogação do material pedagógico existente através da utilização de MDs, pois, de acordo com Gomes (2005, p. 202) é necessário que os OAs sejam descritos segundo um padrão de metadados, possibilitando que os OAs tornem-se flexíveis, reutilizáveis, customizáveis e interoperáveis (características estas que facilitam a sua utilização). (Ministério da Educação, 2007, p. 20).

Nos próximos tópicos serão descritos os mapeamentos realizados para as versões de perfis de MDs criadas para o sistema UNASUS. Foram desenvolvidas duas versões de perfis de MDs para a UNASUS, ambas em parceria com a Universidade Federal de Ciências da Saúde de Porto Alegre (UFCSPA).

\subsection{Padrão de metadados UNASUS - primeira versão}

A primeira versão data de junho de 2011 a qual acabou por ser substituída em setembro do mesmo ano pela segunda versão, que é a mais atual. A primeira versão possuía um total de $50 \mathrm{MDs}$, sendo que, de acordo com o estudo realizado, 39 eram totalmente compatíveis com o padrão OBAA, 7 seriam extensões do OBAA, e 4 MDs novos seriam propostos como incorporação ao próprio padrão OBAA.

Durante o mapeamento entre os dois padrões observou-se que alguns MDs não eram compatíveis com o OBAA, pois tratavam-se de características muito específicas do sistema UNASUS e, desta forma, a proposta foi de extensão e não de incorporação ao padrão, além disso, observou-se que 4 MDs tinham informações de direitos autorais que seriam úteis ao OBAA e por isso foi feita a proposta de incorporá-los ao referido padrão. $\mathrm{O}$ quadro a seguir contém os metadados que são totalmente compatíveis com o OBAA. Detalhes sobre cada um deles podem ser encontrados em Vicari et al. (2009).

\begin{tabular}{|l|l|l|l|}
\hline Item & Metadado UNASUS & Item & Metadado OBAA \\
\hline 1 & Identification & 1 & general \\
\hline 1.1 & unasus.identifier.uri & 4.3 & location \\
\hline 1.2 & unasus.title & 1.2 & title \\
\hline 1.3 & unasus.contributor. $\{$ contrib\} & 2.3 .2 & entity \\
\hline 1.4 & unasus.source & 4.3 & location \\
\hline 1.5 & unasus.language.iso & 1.3 & language \\
\hline 1.6 & unasus.description.abstract & 1.4 & description \\
\hline 1.7 & unasus.subject & 1.5 & keyword \\
\hline 1.8 & unasus.subject. \{taxon $\}$ & 9.4 & keyword \\
\hline
\end{tabular}




\begin{tabular}{|l|l|l|l|}
\hline 1.9 & unasus.structure & 1.7 & structure \\
\hline 1.10 & unasus.aggregationLevel & 1.8 & aggregationLevel \\
\hline 1.11 & unasus.date.issued & 2.3 .3 & date \\
\hline 2 & LifeCycle & 2 & lifeCycle \\
\hline 2.1 & unasus.version & 2.1 & version \\
\hline 2.2 & unasus.status & 2.2 & status \\
\hline 3 & Technical & 4 & techincal \\
\hline 3.1 & unasus.technicalType & 4.4 .1 .1 & type \\
\hline 3.2 & unasus.format.mimetype & 4.1 & format \\
\hline 3.3 & unasus.format.extent & 4.2 & size \\
\hline 4 & Educational & 5 & educational \\
\hline 4.1 & unasus.interactivityType & 5.1 & interactivityType \\
\hline 4.2 & unasus.learningResourceType & 5.2 & learningResourceType \\
\hline 4.3 & unasus.intendedEndUserRole & 5.5 & intendedEndUserRole \\
\hline 4.5 & unasus.audience.context & 5.6 & context \\
\hline 4.6 & unasus.learningContext & 5.6 & context \\
\hline 4.7 & unasus.typicalLearningTime & 5.9 & typicalLearningTime \\
\hline 4.8 & unasus.educacionalDescription & 5.10 & description \\
\hline 4.9 & unasus.instructionalMethod & 8.3 & description \\
\hline 5 & Rights & 6 & rights \\
\hline 5.3 & unasus.rights.license & 6.3 & description \\
\hline 6 & Classification & 9 & classification \\
\hline 6.1 & unasus.classification & 9.2 .1 & source \\
\hline 7 & Relation & 7 & relation \\
\hline 7.1 & unasus.relation.\{relation $\}$ & 7.1 & kind \\
\hline 8 & Control & 2 & lifeCycle \\
\hline 8.1 & unasus.date.accessioned & 2.3 .3 & date - conforme 2.1 - version \\
\hline 8.2 & unasus.date.available & 2.3 .3 & date - conforme 2.1 - version \\
\hline 8.3 & unasus.date.modified & 2.3 .3 & date - conforme 2.1 - version \\
\hline
\end{tabular}

Quadro 1: Metadados UNASUS da versão 1 compatíveis com OBAA

Já as informações a seguir contêm os MDs que serão de uso exclusivo do perfil OBAA/UNASUS, já que são informações muito específicas ao contexto da UNASUS.

- 1.12 unasus.local.issued: Local da publicação original do OA.

- 2.3 unasus.description.sponsorship: Patrocinador do recurso educacional.

- 2.4 unasus.avaliation.\{type\}: Avaliações internas e/ou externas.

- 2.5 unasus.date.valid: Data esperada para que o recurso perca sua validade educacional.

- 3.4 unasus.format.description: Descrição física do recurso educacional.

- 3.5 unasus.technicalRequirement: Requisitos técnicos necessários para visualização do recurso educacional.

- 4.4 unasus.audience.occupation: Ocupação do Público Alvo planejado para 
o recurso.

Além disso, os seguintes metadados foram propostos para serem incorporados ao padrão OBAA.

- 5.1 unasus.rightsHolder: Detentor(es) do direito autoral do recurso educacional.

- 5.2 unasus.rights.type: Tipo de direito autoral.

- 5.4 unasus.rights.dateLicenseExpired: Data em que deixa de ter validade o regime de direitos autorais, no caso deste campo não ser utilizado considerase a validade indeterminada.

- 5.5 unasus.rights.accessRights: Indica se o material oferece risco à população se tiver divulgação ampla.

\subsection{Padrão de metadados UNASUS - segunda versão}

O segundo perfil criado com os metadados da UNASUS possui as seguintes características: total de $51 \mathrm{MDs}$, sendo que 32 são totalmente compatíveis com o padrão OBAA, 16 são extensões do OBAA e 3 MDs serão incorporados ao próprio padrão OBAA. Esta diferença entre a primeira e a segunda versão do perfil, ocorre devido às várias modificações realizadas na organização dos MDs realizadas pela própria UNASUS. Uma nova análise foi necessária para a criação do segundo perfil e o resultado obtido será descrito a seguir.

Da mesma forma como na primeira versão do perfil, vários MDs da UNASUS são compatíveis com o OBAA e podem ter suas informações obtidas em Vicari et al. (2009). O quadro a seguir representa o mapeamento dos MDs compatíveis entre os dois padrões:

\begin{tabular}{|l|l|l|l|}
\hline Item & Metadado UNASUS & Item & Metadado OBAA \\
\hline & classificação & 9 & classification \\
\hline 4.1 & unasus.learningResourceType & 5.2 & learningResourceType \\
\hline & descrição geral & 1 & general \\
\hline 1.2 & dc.title & 1.2 & title \\
\hline & dc.contributor.author & 2.3 .2 & entity \\
\hline 1.4 & unasus.contributor.\{contrib\} & 2.3 .1 & role \\
\hline 2.1 & unasus.version & 2.1 & version \\
\hline 1.6 & dc.language.iso & 1.3 & language \\
\hline 1.13 & dc.date.created & 2.3 .3 & date \\
\hline 1.14 & dc.date.issued & 2.3 .3 & date \\
\hline 1.5 & dc.source & 4.3 & location \\
\hline & descrição educacional & 5 & educational \\
\hline 4.3 & unasus.audience.context & 5.6 & context \\
\hline & unasus.restrictedAccess & 6.2 & copyrightAndOtherRestrictions \\
\hline 4.6 & unasus.educacionalDescription & 5.10 & description \\
\hline
\end{tabular}




\begin{tabular}{|l|l|l|l|}
\hline 4.5 & unasus.typicalLearningTime & 5.9 & typicalLearningTime \\
\hline 1.10 & unasus.structure & 1.7 & structure \\
\hline 1.11 & unasus.aggregationLevel & 1.8 & aggregationLevel \\
\hline 1.9 & unasus.subject.decs & 9.2 .2 .2 & entry \\
\hline 1.8 & dc.subject.keyword & 1.5 & keyword \\
\hline 1.7 & dc.description.abstract & 1.4 & description \\
\hline 7.1 & dc.relation. $\{$ relation $\}$ & 7.2 .1 .2 & entry \\
\hline 7.1 & dc.relation. $\{$ relation $\}$ & 7.1 & kind \\
\hline & direitos autorais e submissão & 6 & rights \\
\hline 5.3 & dc.rights.license & 6.3 & description \\
\hline 3.4 & unasus.technicalRequirement & 4.6 & otherPlatformRequirements \\
\hline 3.1 & dc.format.mimetype & 4.1 & format \\
\hline 3.2 & dc.format.extent & 4.2 & size \\
\hline 8.1 & dc.date.accessioned & 2.3 .3 & date - conforme 2.1 - version \\
\hline 8.2 & dc.date.available & 2.3 .3 & date - conforme 2.1 - version \\
\hline 8.3 & dc.date.modified & 2.3 .3 & date - conforme 2.1 - version \\
\hline 1.1 & dc.identifier.uri & 1.1 .2 & entry \\
\hline
\end{tabular}

Quadro 2: Metadados UNASUS da versão 2 compatíveis com OBAA

Com relação aos MDs de direitos autorais que foram propostos para serem incorporados ao OBAA, permanecem os mesmo descritos na listagem da versão 1 e, da mesma forma descrita anteriormente, alguns MDs específicos do contexto UNASUS serão estendidos ao padrão OBAA, conforme descrito a seguir:

- 6.1 unasus.classification: Classificação temática do recurso educacional no Acervo UNA-SUS.

- $\quad 1.3$ dc.title.alternative: Título alternativo do recurso educacional.

- 2.3 unasus.description.sponsorship: Patrocinador do recurso educacional.

- (sem numeração) dc.mediator: Instituição responsável pela submissão do recurso educacional.

- 1.12 dc.location.country: País de origem do recurso educacional.

- 3.3 unasus.format.description: Descrição das características físicas do recurso educacional.

- 1.15 dc.publisher: Editora responsável pelo recurso educacional caso já tenha sido publicado anteriormente.

- 4.4 unasus.learningContext: Nível educacional para o qual o recurso educacional foi planejado.

- 4.2 unasus.audience.occupation: Ocupação do público-alvo para o qual o recurso educacional foi planejado.

- 2.3 unasus.avaliation.content: Informação sobre a avaliação do recurso educacional, incluindo tipo de avaliação e responsável. Obrigatoriamente devem ser realizadas avaliações de conteúdo, pertinência e midiático- 
pedagógica.

- (sem numeração) unasus.avaliation.relevance: Informação sobre a avaliação do recurso educacional, incluindo tipo de avaliação e responsável. Obrigatoriamente devem ser realizadas avaliações de conteúdo, pertinência e midiático-pedagógica.

- (sem numeração) unasus.avaliation.media-pedagogical: Informação sobre a avaliação do recurso educacional, incluindo tipo de avaliação e responsável. Obrigatoriamente devem ser realizadas avaliações de conteúdo, pertinência e midiático-pedagógica.

- 4.7 dc.instructionalMethod: Recomendações pedagógicas para uso do recurso educacional.

- 2.4 unasus.date.valid: Data de validade do recurso educacional determinada de acordo com seu conteúdo ou seus aspectos educacionais . O não preenchimento significa validade indeterminada.

- (sem numeração) descrição temática: Descrição dos assuntos abordados no recurso educacional

- 6.1 unasus.classification: Classificação temática do recurso educacional no Acervo UNA-SUS.

\section{Considerações Finais}

Este artigo apresentou a proposta de padrão de metadados brasileiro para catalogação de OAs, o OBAA, e a criação de um perfil que possibilita sua utilização na área da saúde. Também foi apresentado como adicionar informações ao perfil através do mecanismo de extensão que o OBAA herda do padrão LOM.

Além disso, é importante que o desenvolvedor do perfil seja conhecedor dos metadados do OBAA também sendo necessária uma análise prévia do outro padrão que servirá de base para a criação do perfil (neste caso o UNASUS), para que os metadados dos dois padrões sejam relacionados corretamente.

Como trabalhos futuros, o perfil de MDs apresentado nesse artigo será utilizado para o desenvolvimento de um repositório de OAs junto a UFCSPA. Repositório semelhante ao apresentado em http://repositorio.portalobaa.org, porém com modificações nos metadados e possíveis alterações de permissões conforme necessidade da instituição. Por fim, esse trabalho serve de base para o desenvolvimento de novos perfis de metadados para outras diferentes áreas de ensino.

\section{Referências Bibliográficas}

BEHAR, PATRICIA ALEJANDRA; GASPAR, MARIA IVONE. Uma perspectiva curricular com base em objetos de aprendizagem. [S.l.: s.n.], 2007. Disponível em: <http://ihm.ccadet.unam.mx/virtualeduca2007/pdf/37-PB.pdf >. Acesso em: 28 out. 2012. 
DURASPACE. DSpace Official Website. [S.l.: s.n.]. Disponível em: <http://www.dspace.org/>. Acesso em: 22 out. 2012.

GOMES, SIMONE ROCHA et al. Objetos de Aprendizagem Funcionais e as Limitações dos Metadados Atuais. [S.l.: s.n.], 2005. Disponível em: <http://brie.org/pub/index.php/sbie/article/download/406/392>. Acesso em: 27 out. 2012.

IEEE COMPUTER SOCIETY. IEEE P1484.12.3, Draft 8 Draft Standard for Learning Technology - Extensible Markup Language (XML) Schema Definition Language Binding for Learning Object Metadata. Nova Iorque - NY, 2005. Disponível em: <http://ltsc.ieee.org/wg12/files/IEEE_1484_12_03_d8_submitted.pdf>. Acesso em: 20 out. 2012.

IMS GLOBAL LEARNING CONSORTIUM, INC. IMS AccessForAll Meta-data Information Model. [S.1.: s.n.], 2004. Disponível em: <http://www.imsglobal.org/accessibility/accmdv1p0/imsaccmd_infov1p0.html>. Acesso em: 20 out. 2012.

MCPARLAND, ANDREW. TV-Anytime Website. [S.1.: s.n.], 2005. Disponível em: <http://www.tv-anytime.org/>. Acesso em: 29 out. 2012.

MINISTÉRIO DA EDUCAÇÃO. Objetos de aprendizagem: Uma proposta de recursos pedagógicos. Brasília, DF: Secretária da Educação a Distância, 2007. 154 p.

UNASUS. Portal Universidade Aberta do SUS. Brasília, 2011. Disponível em: <http://www.unasus.gov.br/node/1>. Acesso em: 22 out. 2012.

VICARI, ROSA MARIA et. al. Relatório Técnico RT-OBAA-01: Proposta de Padrão para Metadados de Objetos de Aprendizagem Multiplataforma. Porto Alegre: UFRGS, 2009 a. 78 p. Disponível em: <http://www.portalobaa.org/padrao-obaa/relatoriostecnicos/relatorio-final-proposta-obaa-finep/RT-OBAA-01.pdf/at_download/file>. Acesso em: 15 out. 2012.

VÆRVÅGEN, TORMOD; DAHL, GUNNAR. TVAMainType - XSD Representation. Noruega, $2007 . \quad$ Disponível em: <http://gluon.nrk.no/gluondok2/gluon2_TVAMainType.html>. Acesso em: 20 out. 2012.

XAVIER OCHOA et. al. On the use of Learning Object Metadata: the GLOBE experience. Heverlee, Belgica, 2009. Disponível em: $<$ http://ariadne.cti.espol.edu.ec/xavier/papers/Ochoa-ECTEL2011.pdf >. Acesso em: 20 out. 2012. 